\title{
Looking for GRB progenitors
}

\author{
Krzysztof Belczyński ${ }^{1}$, Tomasz Bulik ${ }^{1}$ and Bronisław Rudak ${ }^{2,3}$
}

\author{
${ }^{1}$ Nicolaus Copernicus Astronomical Center, Bartycka 18, 00-716 Warszawa,Poland \\ 2 Nicolaus Copernicus Astronomical Center, Rabiańska 8, 87-100 Toruń,Poland \\ 3 Toruń Center for Astronomy, Nicolaus Copernicus University, Gagarina 11, 87-100 Toruń, \\ Poland
}

\begin{abstract}
Using stellar binary population synthesis code we calculate the production rates and lifetimes of several types of possible GRB progenitors. We consider mergers of double neutron stars, black hole neutron stars, black hole white dwarfs and helium star mergers. We calibrate the results with the measured star formation rate history. We discuss the viability of each GRB model, and alternatively assuming that all bursts are connected with one model we constrain the required collimation of GRBs. We also show the importance of widely used evolutionary parameters on the merger rates of calculated binary populations.
\end{abstract}

\section{INTRODUCTION}

It is surprising how little we know of GRB progenitors when we consider how much work is devoted to the subject. Different objects and models of progenitors were proposed, some already forgotten while others still being intensively studied. Considering the diversity of gamma ray bursts it is probable that they come from different types of astronomical objects, and this should promote the work on different types of proposed progenitors. Recently much of the weight was placed on collapsars, and connection between supernovae and GRBs, although other models, as compact object mergers, are still on the stage.

Compact object binaries have recently drawn much attention in the astronomical community. Several models of compact object binaries were proposed as GRBs progenitors, namely: double neutron star mergers [11,18], black hole - neutron star mergers [9], black hole - white dwarf mergers [8] and helium star mergers [6]. Mergers of compact object binaries are also most often considered sources of gravitational waves. As gravitational wave detectors, LIGO and VIRGO, will soon be operational, the question of these merger rates arises [1].

To predict compact object merger rates we use Monte Carlo simulations to produce populations of different compact object binaries. In this approach one generates massive binaries at ZAMS and evolves them through consecutive stages of 
single and binary star evolution, which may eventually lead to formation of a compact object binary. Final synthesis of large ensembles of compact object binaries allows then statistical studies and calculation of expected merger rates.

Several compact object merger rates estimates $[12,13,21,17,2,3,7]$ have been already published. However, calculations of this rates are based on many assumptions and use parameters with very uncertain values. Whereas the evolution of single stars is reasonably well known [5], the distributions of initial binary conditions as well as some aspects of binary star evolution are uncertain. Finally the population synthesis codes must deal with uncertainties in supernova explosion mechanisms, and in particular with the value of the kick a neutron star (or a black hole) receives at birth, and the mass of formed in the explosion compact object [3]. Previous population synthesis calculations concerned double neutron stars and only few dealt with neutron star black hole binaries. So far only in one case [7] all kinds of the proposed GRBs compact object binary progenitors were considered. Our present contribution follows the same line of work, although we use a different population synthesis code and we would like to communicate our first results here. Preliminary comparison shows striking similarities of the results, which taking into account different codes, points toward robustness of the population synthesis method in spite of many uncertainties involved in the calculations.

\section{THE MODEL}

Our evolutionary code is primarily based on the prescriptions given by [20] and [5], but we use the number of many revised or newly developed specific evolutionary prescriptions from $[4,16,15,14]$. In our calculation of single and binary evolution we include stellar winds (normal and LBV wind), magnetic breaking, quasi-dynamic mass transfer, common envelope evolution, hyper-accretion onto compact objects, detailed supernova explosion treatment and gravitational wave energy loss in compact object binaries. We start the evolution of a given binary when both components are at ZAMS, then each star evolves through different stages of its life depending on its mass: main sequence, Hertzsprung gap, red giant branch, horizontal branch, asymptotic giant branch, and either supernova explosion which leads to formation of a neutron star (or a black hole) or a phase of enhanced mass loss and formation of a white dwarf. During each stage of the binary evolution the components may interact, which changes the consecutive component's evolution (either through rejuvenation, stripping the component off its outer layers, or even by swallowing one component by the other). Interaction of binary components may lead to one of the proposed GRB progenitors, the helium star merger, when the compact object is engulfed in giant's envelope. If there is not enough orbital energy to eject the giant's envelope then the compact object spirals in through the giant's envelope finally merging with the giant's core, which can be torn out in the process, and form an accretion disc around a compact object which is then already a black hole, due to the accretion during spiral in. 


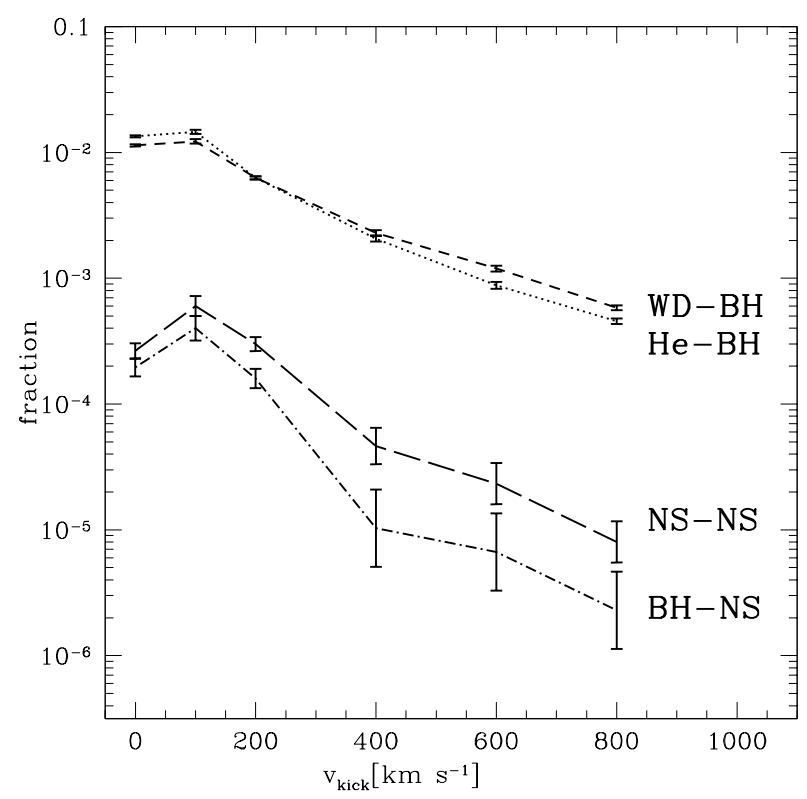

FIGURE 1. Relative production rates of different GRB progenitor types that merge within the Hubble time.

Other systems follow their evolutionary paths, and we collect the informations on the types of compact object binaries that are proposed for GRBs progenitors: double neutron stars, black hole neutron stars and black hole white dwarf systems. These systems interact only due to gravitational energy wave loss, finally merging and forming a black hole with massive thick accretion disc which presumably may lead to a gamma ray burst.

\section{RESULTS}

In Fig. 1 we show the relative numbers of 4 different GRB progenitor types that merge within the Hubble time (15 Gyrs) as a function of the width of the distribution from which we draw kick velocity a compact object receives in a supernova explosion. Two things are clearly seen; first the number of WD-BH binaries and Helium mergers (He- $\mathrm{BH})$ is about the same and is more then an order of magnitude greater then the number of NS-NS and BH-NS binaries. Second, the number of a given progenitor type falls off approximately exponentially with the kick velocity. Relative production rates may be calibrated (eg. see eq. 14 in [2]). For example, assuming the width of kick velocity, let say, $\mathrm{v}_{k i c k}=200 \mathrm{~km} \mathrm{~s}^{-1}$ yields: 1 merging event per Milky Way like galaxy per Myr for BH-NS systems, 3 events for NS-NS binaries and 60 for WD-BH and Helium mergers.

In Fig. 2 we show the cumulative rate of different merging events. We have combined our relative numbers for different progenitors with the star formation rate function $[10,19]$, and taking into account the evolutionary time delay of a 


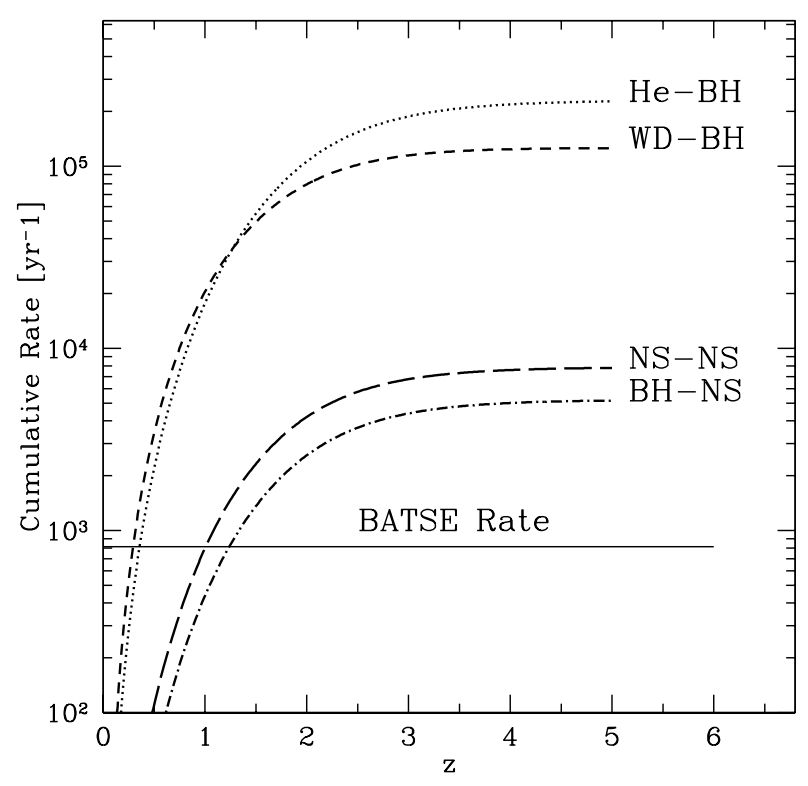

FIGURE 2. Cumulative merger rates for different GRB progenitor types as a function of redshift.

given merging event we integrated our production rates to get the merger rates as a function of redshift. In this example calculation we assumed $\Omega_{M}=1.0, \Omega_{\Lambda}=0.0$, and the Hubble constant $H_{0}=65 \mathrm{~km} \mathrm{~s}^{-1} \mathrm{Mpc}^{-1}$. We used the kicks drawn from the distribution which is a weighted sum of two Gaussians: 80 percent with the width of $200 \mathrm{~km} \mathrm{~s}^{-1}$ and 20 percent with the width $800 \mathrm{~km} \mathrm{~s}^{-1}$.

In Fig. 2 we show also BATSE gamma ray burst detection rate corrected for the sky exposure. Comparison of the cumulative distributions for different progenitor types with he BATSE rate shows that if any of the progenitor types included here was to reproduce the BATSE rate, then we should not see GRBs from redshifts greater then unity! Of course this is not the case, as GRBs with higher redshifts were observed. However, we haven't yet introduced the collimation factor into our results, presented in Fig. 2, which certainly will lower down our predicted rates. To lower down our calculated rates to the BATSE rate, for average GRBs redshift of about 2, we would need the collimation of about $4^{\circ}$ for BH-WD and Helium mergers and about $12^{\circ}$ for BH-NS and NS-NS mergers.

As seen in Fig. 2 the curves flatten out for high redshifts $(\mathrm{z} \geq 5)$. In other words we do not expect binary mergers at high redshifts. This is a combined effect of the star formation rate function we have used, which falls down for high redshifts and of the non zero lifetimes of progenitors, prior to the final merging event. Each of our binaries needs a specific time to evolve into a compact object binary $\left(\mathrm{t}_{\text {evol }}\right)$ and then needs time to merge due to gravitational wave energy losses $\left(t_{\text {merger }}\right)$. This times are non negligible and are specific for each group of proposed binary GRBs progenitors. For our sample of binaries we found that $t_{\text {life }}\left(t_{\text {evol }}+t_{\text {merger }}\right)$ are, for 
NS-NS: $\sim 10^{7}-10^{12} \mathrm{yrs}$, for BH-NS: $\sim 10^{7}-10^{10} \mathrm{yrs}$, for BH-He: $\sim 10^{6}-10^{9} \mathrm{yrs}$, for BH-WD: $~ 10^{7}-10^{12}$ yrs.

\section{CONCLUSIONS}

- GRB binary progenitor production rates fall off exponentially with width of natal kick velocity distribution.

- Evolutionary times can not be neglected in computations of GRB's progenitor rates.

- Assuming that all GRB's are connected to NS-NS or BH-NS binaries, the collimation must be of order $\sim 3 \times 10^{-2}\left(12^{\circ}\right)$. If we assume that all GRB's result from binary mergers, then the population is dominated by $\mathrm{BH}-\mathrm{WD}$ and $\mathrm{BH}-\mathrm{He}$ star mergers, and the collimation must be of order $\sim 3 \times 10^{-3}\left(4^{\circ}\right)$.

- We do not expect binary mergers at high redshifts $(\mathrm{z} \geq 5)$.

Acknowledgments. We acknowledge the support of the following grants KBN2P03D01616, KBN-2P03D00415, KBN2P03D02117.

\section{REFERENCES}

1. Allen B. et al., Phys. Rev. Lett. 83, 1498 (1999).

2. Belczynski K. and Bulik T., A\&A 346, 91 (1999).

3. Belczynski K., Bulik T. and Zbijewski W., A $\& A$ accepted, astro-ph/9911435 (1999).

4. Bethe H.A. and Brown G.E., ApJ 506, 780 (1998).

5. Eggleton P.P., Tout C.A. and Fitchett M.J., ApJ 347, 998 (1989).

6. Fryer C.L. and Woosley S., ApJ Lett. 502, L9 (1998).

7. Fryer C.L., Woosley S. and Hartmann D.H., ApJ submitted, astro-ph/9904122 (1999).

8. Fryer C.L., Woosley S., Herant M. and Davies M.B., ApJ 520, 650 (1999).

9. Lee W.H. and Kluźniak W., Acta Astronomica 45, 705 (1995).

10. Madau P. et al., MNRAS 283, 1388 (1996).

11. Meszaros P. and Rees M.J., ApJ 476, 232 (1997).

12. Narayan R., Piran T. and Shemi A., ApJ Lett. 379, L17 (1991).

13. Phinney E.S., ApJ Lett. 380, L17 (1991).

14. Podsiadlowski P., Joss P.C. and Hsu J.J.L., ApJ 391, 246 (1992).

15. Pols O.R. and Marinus M., A $\& A$ 288, 475 (1994).

16. Portegies Zwart S.F. and Verbunt F., $A \& A$ 309, 179 (1996).

17. Portegies Zwart S.F. and Spreeuw H.N., $A \mathscr{E} A$ 312, 670 (1996).

18. Ruffert M., Janka H.T., Takahashi K. and Schaefer G., A $E A$ 319, 122 (1997).

19. Totani T.,ApJ Lett. 486, L71 (1997).

20. Tout C.A., Aarseth S.J., Pols O.R. and Eggleton P.P., MNRAS 291, 732 (1997).

21. Tutukov A.V. and Yungelson L.R., MNRAS 260, 675 (1993). 